\title{
Principal axes for stochastic dynamics
}

\author{
V.V. Vasconcelos, ${ }^{1}$ F. Raischel, ${ }^{2}$ M. Haase,${ }^{3}$ J. Peinke, ${ }^{4}$ M. Wächter, ${ }^{4}$ P.G. Lind,${ }^{1,2}$ and D. Kleinhans ${ }^{5,6}$ \\ ${ }^{1}$ Physics Department, Faculty of Sciences, University of Lisbon, 1649-003 Lisbon, Portugal \\ ${ }^{2}$ Center for Theoretical and Computational Physics, University of Lisbon, Av. Prof. Gama Pinto 2, 1649-003 Lisbon, Portugal \\ ${ }^{3}$ Institute for High Performance Computing, University of Stuttgart, Nobelstr. 19, DE-70569 Stuttgart, Germany \\ ${ }^{4}$ Institute of Physics, University of Oldenburg, DE-26111 Oldenburg, Germany \\ ${ }^{5}$ Institute for Marine Ecology, University of Gothenburg, Box 461, SE-405 30 Göteborg, Sweden \\ ${ }^{6}$ Institute of Theoretical Physics, University of Münster, DE-48149 Münster, Germany
}

\begin{abstract}
We introduce a general procedure for directly ascertaining how many independent stochastic sources exist in a complex system modeled through a set of coupled Langevin equations of arbitrary dimension. The procedure is based on the computation of the eigenvalues and the corresponding eigenvectors of local diffusion matrices. We demonstrate our algorithm by applying it to two examples of systems showing Hopf-bifurcation. We argue that computing the eigenvectors associated to the eigenvalues of the diffusion matrix at local mesh points in the phase space enables one to define vector fields of stochastic eigendirections. In particular, the eigenvector associated to the lowest eigenvalue defines the path of minimum stochastic forcing in phase space, and a transform to a new coordinate system aligned with the eigenvectors can increase the predictability of the system.
\end{abstract}

PACS numbers: 02.50.Ga, 02.50.Ey, 89.65.Gh, 92.70.Gt

Keywords: Stochastic Systems, Langevin equation, Predictability

\section{INTRODUCTION}

When dealing with measurements on complex systems it is typically difficult to find the optimal set of variables to describe their evolution, which provides the best opportunities for understanding and predicting the system's behavior.

Recently a framework for analyzing measurements on stochastic systems was introduced [1, 2]. This framework is based on the assumption that the measured properties evolve according to a deterministic drift and stochastic fluctuations due to the interactions with the internal degrees of freedom or the environment. These fluctuations are globally ruled by some specific stochastic forcing inherent to the signal evolution itself, and therefore cannot be separated from the measured variables. Whether they are due to quantum uncertainties or a limited knowledge of the state of the system, they are fundamental for a complete description of a complex system. Several works in this scope have already shown the advantage of this approach, ranging from the description of turbulent flows [1] and climate indices [3] to the evolution of stock markets [4] and oil prices [5], just to mention some. In the course of time, several improvements to the method were proposed concerning its robustness with respect to finite sampling effects and measurement noise [6-11].

However, a natural question arises when dealing simultaneously with several variables: is it possible to find non-trivial functions of measured properties for which the stochastic fluctuation can be neglected? This would mean that it should be possible to decrease the number of stochastic variables needed to describe the system.

In this paper we will follow this question up in detail and show, that by accessing the eigenvalues of the diffusion matrices comprehending the measured properties it is possible to derive a path in phase space through which the deterministic contribution is enhanced. As a direct application, our procedure allows for the determination of the number of independent sources of stochastic forcing in a system described by an arbitrarily large number of properties.

A pictorial example is as follows. Consider a professional archer trying to aim at a target in a succession of shots. If the archer holds the bow without any support, one expects that the set of trials is distributed around the center according to a radially symmetric Gaussian. Hence deviations have the same amplitudes in all directions. However, if the archer lies on the floor, the vertical direction will be more confined than the horizontal. In this case we expect a smaller variance of deviations in the vertical direction than in the horizontal one. If the archer lies on an inclined plane the most confined and less confined directions will have a certain slope related to the plane inclination. Regarding the variance in space as a particular representation of the diffusion matrix, we can now think in more complex systems where at each point in phase space fluctuations can be defined through their local diffusion matrices. The study of its eigenvalues and eigenvectors is the scope of the present paper.

We start in Sec. II by defining our system of variables mathematically and by describing the standard approach to estimate its dynamics from measured data as introduced in Ref. [1, 2], where a particular emphasis will be given to the diffusive terms. Then the general eigenvalue problem is introduced in the role of the local eigenvalues of diffusion matrices, which exhibits the framework for the present work. We show that at each point of phase space the eigenvectors of the diffusion matrix are tangent to new coordinate lines, one of them corresponding to the lowest eigenvalue, thus indicating the direction towards which fluctuations are - at least partially - suppressed. In Sec. III we demonstrate our approach on two examples from Hopf-bifurcation systems with stochastic forcing. Section IV closes the paper with discussion and conclusions. 


\section{DEFINING STOCHASTIC EIGENDIRECTIONS}

We consider an $N$-dimensional Langevin process $\mathbf{X}=$ $\left(X_{1}(t), \ldots, X_{N}(t)\right)$ whose probability density functions (PDFs) $f(\mathbf{X}, t)$ evolve according to the Fokker-Planck equation (FPE) [12, 13]

$$
\begin{aligned}
\frac{\partial f(\mathbf{X}, t)}{\partial t}= & -\sum_{i=1}^{N} \frac{\partial}{\partial x_{i}}\left[D_{i}^{(1)}(\mathbf{X}) f(\mathbf{X}, \mathbf{t})\right] \\
& +\sum_{i=1}^{N} \sum_{j=1}^{N} \frac{\partial^{2}}{\partial x_{i} \partial x_{j}}\left[D_{i j}^{(2)}(\mathbf{X}) f(\mathbf{X}, t)\right]
\end{aligned}
$$

The functions $D_{i}^{(1)}$ and $D_{i j}^{(2)}$ are called the Kramers-Moyal or the drift and diffusion coefficients and are defined as

$$
\mathbf{D}^{(k)}(\mathbf{X})=\lim _{\Delta t \rightarrow 0} \frac{1}{\Delta t} \frac{\mathbf{M}^{(k)}(\mathbf{X}, \Delta t)}{k !},
$$

where $\mathbf{M}^{(k)}$ are the first and second conditional moments $(k=1,2)$. Here we assume that the underlying process is stationary and therefore both drift and diffusion coefficients do not explicitly depend on time $t$. Conditional moments can be directly derived from the measured data as [2, 7]:

$$
\begin{aligned}
& M_{i}^{(1)}(\mathbf{X}, \Delta t)=\left\langle Y_{i}(t+\Delta t)-Y_{i}(t) \mid \mathbf{Y}(t)=\mathbf{X}\right\rangle \\
& M_{i j}^{(2)}(\mathbf{X}, \Delta t)= \\
& \left\langle\left(Y_{i}(t+\Delta t)-Y_{i}(t)\right)\left(Y_{j}(t+\Delta t)-Y_{j}(t)\right) \mid \mathbf{Y}(t)=\mathbf{X}\right\rangle
\end{aligned}
$$

Here $\mathbf{Y}(t)=\left(Y_{1}(t), \ldots, Y_{N}(t)\right)$ exhibits the $N$-dimensional vector of measured variables at the $t$ and $\langle\cdot \mid \mathbf{Y}(t)=\mathbf{X}\rangle$ symbolizes a conditional averaging over the entire measurement period, where only measurements with $\mathbf{Y}(t)=\mathbf{X}$ are taken into account [28]. $\mathbf{D}^{(1)}$ is the drift vector and $\mathbf{D}^{(2)}$ the diffusion matrix. Notice that $\mathbf{Y}(t)=\mathbf{X}$ is considered to be true in a vicinity of $\mathbf{X}$ due to the observational limitations. This vicinity must be small and such that the conditional moments do not change abruptly for small $\Delta t$, making $\mathbf{D}^{(1)}$ and $\mathbf{D}^{(2)}$ continuous.

Associated with the FPE (1) is a system of $N$ coupled ItôLangevin equations, which can be written as [12, 13]

$$
\frac{d \mathbf{X}}{d t}=\mathbf{h}(\mathbf{X})+\mathbf{G}(\mathbf{X}) \boldsymbol{\Gamma}(t)
$$

Here $\boldsymbol{\Gamma}(t)$ is a set of $N$ normally distributed random variables fulfilling

$$
\left\langle\Gamma_{i}(t)\right\rangle=0, \quad\left\langle\Gamma_{i}(t) \Gamma_{j}\left(t^{\prime}\right)\right\rangle=2 \delta_{i j} \delta\left(t-t^{\prime}\right) \quad,
$$

that drives the stochastic evolution of $\mathbf{X}$. The vectors $\mathbf{h}$ and the matrices $\mathbf{G}=\left\{g_{i j}\right\}$ for all $i, j=1, \ldots, N$ are connected to the local drift and diffusion function through

$$
\begin{aligned}
& D_{i}^{(1)}(\mathbf{X})=h_{i}(\mathbf{X}) \quad \text { and } \\
& D_{i j}^{(2)}(\mathbf{X})=\sum_{k=1}^{N} g_{i k}(\mathbf{X}) g_{j k}(\mathbf{X})
\end{aligned}
$$

Such methodology is applicable under certain conditions, namely the markovian nature of the underlying process. Further, the FPE is only valid as long as $D^{(k)} \sim 0$ for $k \geq 3$. In case the stochastic force has zero average and is Gaussian and $\delta$-correlated a Langevin differential equation is obtained. In case the stochastic forces $\Gamma$ not Gaussian $\delta$-correlated, obtaining a stochastic equation is still possible but with stochastic forcing characterized by a Lévy-stable distribution [2, 7]. While the FPE describes the evolution of the joint distribution of the $N$ variables statistically, the system of Langevin equations in (4) models individual stochastic trajectories of the system. In Eq. (4) the term $\mathbf{h}(\mathbf{X})$ contains the deterministic part of the macroscopic dynamics, while the functions $\mathbf{G}(\mathbf{X})$ account for the amplitudes of the stochastic forces mirroring the different sources of fluctuations due to all sorts of microscopic interactions within the system. Here we should point out that we consider stationary processes, otherwise ensemble averages have to be taken[2]. Since $D^{(1)}$ and $D^{(2)}$ are considered to be continuous, the conditional moments should be differentiable (see Eq. (2)), which means that in a sufficiently small time interval the dependence of the conditional moments on the time increment is linear.

The conditional moments in Eq. (3) are computed directly from data time series and are typically linear functions of $\Delta t$ for sufficiently small $\Delta t[2,7]$. Then, through the limit in Eq. (2), both $D_{i}^{(1)}$ and $D_{i j}^{(2)}$ are determined. When dealing

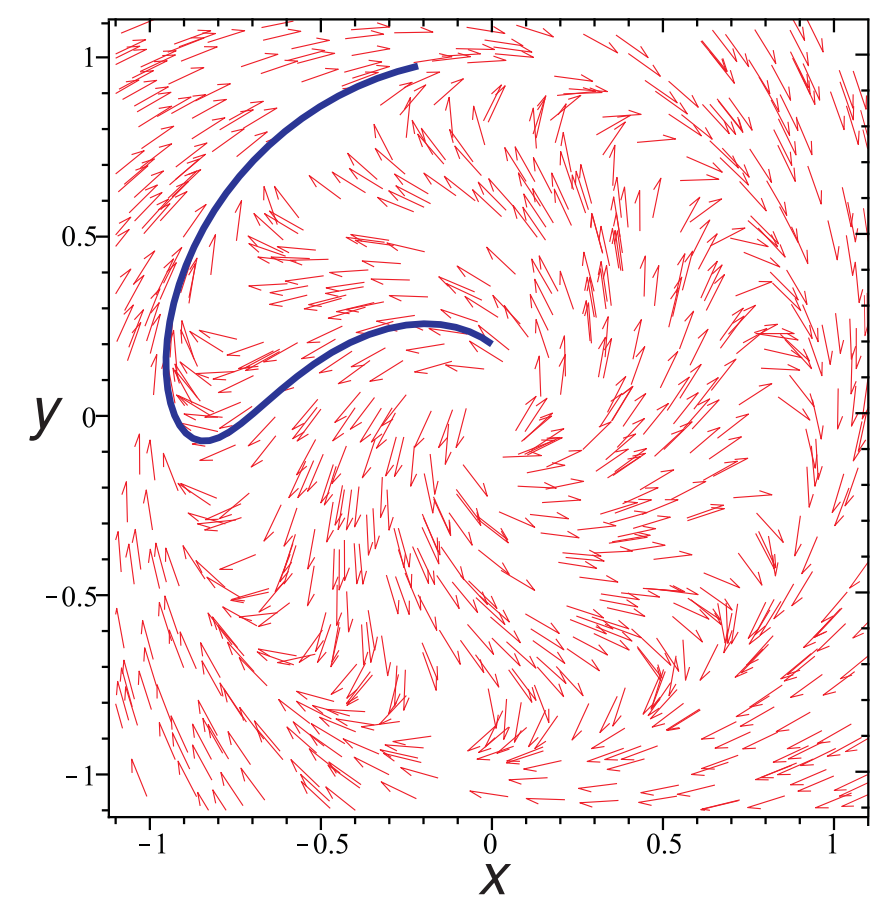

FIG. 1: (Color online) Stream plot of the system in Eq. 8) with no stochasticity $\left(\mathbf{D}^{(2)} \equiv 0\right)$, i.e. $k_{1}=k_{2}=0$, where the arrows formally represent the drift vector of synthetic data extracted from direct simulation of Eq. (8). The solid line represents a partial trajectory. Trajectories diverge from the unstable fixed point $r=0$ and converge to the limit cycle $r=1$. 
(a)

(b)

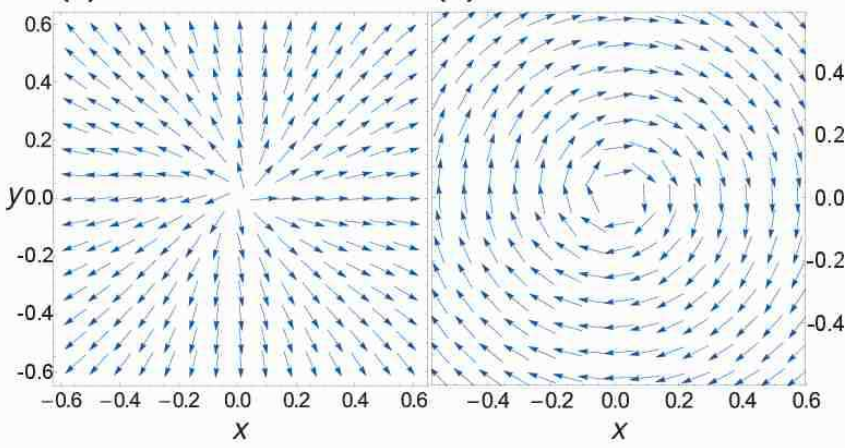

(c)

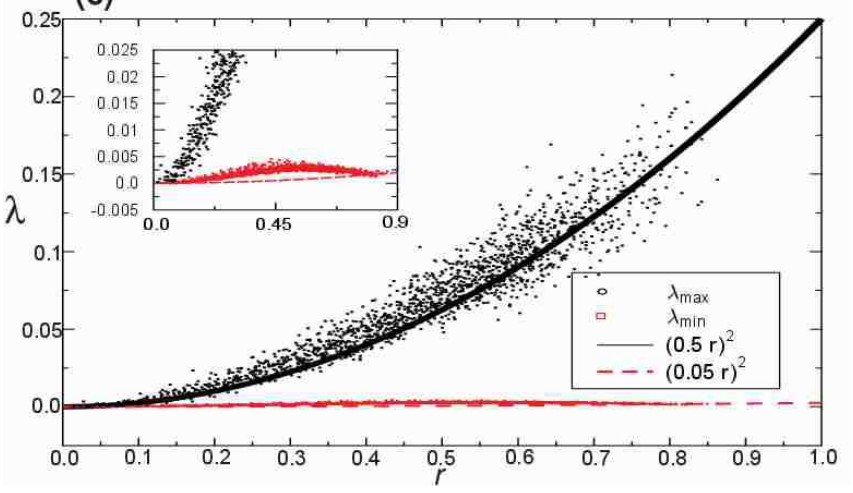

FIG. 2: (Color online) Principal axis of the diffusion matrix. Field of (a) largest and (b) smallest eigenvectors for the diffusion matrix corresponding to Eq. 8 together with (c) the eigenvalues $\lambda$ as a function of $r$ and (inset) a close-up around $\lambda \sim 0$ (see text). The largest eigenvalue is associated with the radial direction while the smallest one corresponds to the angular one. Here $k_{1}=0.5, k_{2}=$ $0.05, \alpha=0.7475 \approx\left\langle r^{2}\right\rangle, t_{0}=0, r_{0}=1.01$ and $\theta_{0}=0$. The analysis was performed from $10^{7}$ data points extracted by integration of Eq. 8] with integration step of $\Delta t=10^{-4}$.

with multidimensional systems which have associated, at least a priori, several independent sources of stochastic fluctuations, the observation time may need to be considerably large, to sample in sufficient detail the system dynamics. Typically, data sets should be not only large but also stationary. Though, recently new developments to such methods were done to suit more general situations were time series are short and nonstationary[27].

The $N \times N$ matrix $\mathbf{G}$ cannot be uniquely determined from the symmetric diffusion matrix $\mathbf{D}^{(2)}$ for $N \geq 2$, because the number of unknown elements in $\mathbf{G}$ exceeds the number of known elements in $\mathbf{D}^{(2)}$ leading to $N^{2}-\frac{1}{2} N(N+1)=$ $\frac{1}{2} N(N-1)$ free parameters. However, a simple method to obtain $\mathbf{G}$ from $\mathbf{D}^{(2)}$ is the following. Due to its symmetry and positive semi-definiteness the diffusion matrix $\mathbf{D}^{(2)}$ has only real, non-negative eigenvalues $\lambda_{i}(i=1, \ldots, N)$. Therefore an orthogonal transformation $\mathbf{U}$ can be found that diagonalizes $\mathbf{D}^{(2)}$, i.e. $\mathbf{U}^{T} \mathbf{D}^{(2)} \mathbf{U}=\operatorname{diag}\left(\lambda_{1}, \ldots, \lambda_{\mathrm{N}}\right)$. Taking the positive root of the eigenvalues and transforming it back we arrive at $g_{i j}=\left(\sqrt{\mathbf{D}^{(2)}}\right)_{i j}$ where the symbolic notation
$\sqrt{\mathbf{D}^{(2)}}=\mathbf{U} \operatorname{diag}\left(\sqrt{\lambda_{1}}, \ldots, \sqrt{\lambda_{\mathrm{N}}}\right) \mathbf{U}^{T}$ is used for simplicity. General forms of $\mathbf{G}$ can be constructed by multiplication of $\sqrt{\mathbf{D}^{(2)}}$ with arbitrary orthogonal matrices. For our considerations the simple version is sufficient [12].

Since $\mathbf{h}$ and $\mathbf{G}$ are functions of the variables $\mathbf{X}$ and are numerically determined on a $n_{1} \times \ldots \times n_{N}$ mesh of points in phase space, one can always define at each mesh point the $N$ eigenvalues and corresponding eigenvectors of the matrix $\mathbf{G}(\mathbf{X})$. This analysis provides information about the stochastic forcing acting on the system and was already applied to a two-dimensional sub-critical bifurcation [15] and to the analysis of human movements [16].

In general the eigenvalues indicate the amplitude of the stochastic force and the corresponding eigenvector indicates the direction toward which such force acts. Even more interesting features, however, can be extracted from the eigenvalues and eigenvectors.

To each eigenvector of the diffusion matrix we can associate one independent source of stochastic forcing $\Gamma_{i}$. In this scope, the eigenvectors can be regarded as defining principal axes for stochastic dynamics. For instance, the vector field aligned at each mesh point to the eigenvector associated to the smallest eigenvalue of matrix $\mathbf{G}$ defines the paths in phase space towards which the fluctuations are minimal. Furthermore, if the corresponding eigenvalues are very small compared to all the other ones at the respective mesh points, the corresponding stochastic forces can be neglected and the system has only $N-1$ independent stochastic forces. In this case the problem can be reduced in one stochastic variable by an appropriate transformation of variables, since the eigenvectors in one coordinate system are the same as in another one (see Append. A.

These are the central ideas of our study which we next apply to an analytical example, namely the Hopf-bifurcation.

\section{THE HOPF-BIFURCATION SYSTEM WITH STOCHASTIC FORCING: AN ANALYTICAL EXAMPLE}

In what follows we consider the dynamical system

$$
\begin{aligned}
& \frac{d r}{d t}=r\left(1-r^{2}\right)+k_{1} r \Gamma_{1} \\
& \frac{d \theta}{d t}=\alpha-r^{2}+k_{2} \Gamma_{2}
\end{aligned}
$$

describing the evolution of the radial and azimuthal coordinates of a particle moving in two-dimensional space, with $\alpha, k_{1}$ and $k_{2}$ being constants. $\Gamma_{1,2}$ are the stochastic forces, which are Gaussian distributed and $\delta$-correlated. For $k_{1}=$ $k_{2}=0$ the system for $\alpha>0$ has an unstable fixed point at $r=0$ and a stable limit cycle at $r=1$, as sketched in Fig. 1. Equation (8b) uses $\alpha-r^{2}$ instead of the usual $1+r^{2}$ [17] in order to be able to avoid the systematic increase of $\theta$ in time. For this reason we chose $\alpha=\left\langle r^{2}\right\rangle$.

Introducing non-zero stochastic terms $k_{1}, k_{2} \neq 0$ for independent stochastic forces, $\Gamma_{1}$ and $\Gamma_{2}$, one has diagonal diffusion matrices with two eigenvalues, $\left(k_{1} r\right)^{2}$ and $k_{2}^{2}$ at each 


\begin{tabular}{|c||c|c|c|c|c|c|c|c|c|c|}
\hline \multirow{10}{*}{$\begin{array}{c}\text { Kramer-Moyal } \\
\text { functions }\end{array}$} & \multicolumn{10}{||c|}{ Coefficients for } \\
\cline { 2 - 12 } & 1 & $x$ & $x^{2}$ & $x^{3}$ & $y$ & $y^{2}$ & $y^{3}$ & $x y$ & $x^{2} y$ & $x y^{2}$ \\
\hline$\tilde{D}_{1}^{(1)}$ & -0.009 & 1.00 & 0.077 & -1.01 & 0.820 & -0.012 & 1.26 & 0.014 & 1.23 & 1.24 \\
\hline$\tilde{D}_{2}^{(1)}$ & -0.034 & 0.761 & 0.133 & -1.09 & 0.99 & 0.036 & -1.22 & 0.101 & -1.00 & -1.21 \\
\hline$\tilde{D}_{11}^{(2)}$ & 0.005 & -0.008 & 0.262 & $\sim 0$ & 0.001 & 0.013 & $\sim 0$ & $\sim 0$ & $\sim 0$ & $\sim 0$ \\
\hline$\tilde{D}_{12}^{(2)}=\tilde{D}_{21}^{(2)}$ & $\sim 0$ & -0.003 & 014 & $\sim 0$ & -0.004 & -0.011 & $\sim 0$ & 0.255 & $\sim 0$ & $\sim 0$ \\
\hline$\tilde{D}_{22}^{(2)}$ & 0.005 & $\sim 0$ & -0.01 & $\sim 0$ & -0.006 & 0.255 & $\sim 0$ & $\sim 0$ & $\sim 0$ & $\sim 0$ \\
\hline
\end{tabular}

TABLE I: Coefficients of quadratic forms describing the surfaces in Fig. 4

(a)

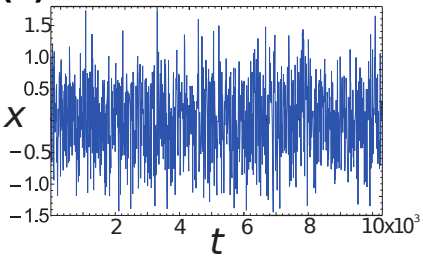

(b)

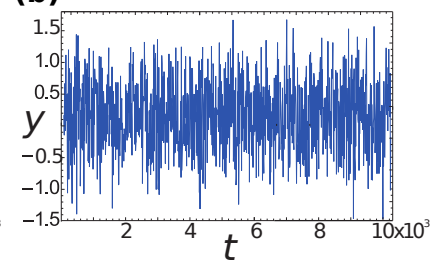

FIG. 3: (Color online) Time series of (a) $x(t)=r(t) \cos \theta(t)$ and (b) $y(t)=r(t) \sin \theta(t)$ where $r$ and $\theta$ are taken from the integration of Eq. 8) for the same conditions as in Fig. 2

point $(r, \theta)$ corresponding to the radial and angular eigendirections respectively.

Since the eigenvectors do not change when changing the coordinate system (see Append. A], "mixing" the radial and angular coordinates by e.g. changing to Cartesian should yield the same principal axis. We, therefore, now repeat our analysis for transformed variables $x=r \cos \theta$ and $y=r \sin \theta$.

Using Itô's formulation for the transformation from polar to Cartesian coordinates [18], the Langevin system can be defined through the drift and diffusion coefficients in transformed coordinates, $\tilde{h}_{i}(x, y), \tilde{g}_{i j}(x, y)$ (see Append. A):

$$
\begin{aligned}
\tilde{h}_{i} & =\left(h_{1} / r-g_{22}^{2}\right) Y_{i}+(-1)^{i} h_{2}\left(Y_{1} \delta_{2 i}+Y_{2} \delta_{1 i}\right)(9 \mathrm{a}) \\
\tilde{g}_{i j} & =\frac{g_{1 j}}{r}\left(Y_{1} \delta_{i 1}+Y_{2} \delta_{i 2}\right)-g_{2 j}\left(Y_{1} \delta_{2 i}+Y_{2} \delta_{1 i}\right)(9 \mathrm{~b})
\end{aligned}
$$

with $i, j=1,2$ and $\left(Y_{1}, Y_{2}\right)=(x, y)$. Thus, from $\mathbf{G}$ in the polar coordinate system, Eq. (9b) yields $\tilde{\mathbf{G}}$ for which the diffusion matrix $\tilde{\mathbf{D}}^{(2)}=\tilde{\mathbf{G}} \tilde{\mathbf{G}}^{T}$ reads

$$
\tilde{\mathbf{D}}^{(2)}=\left[\begin{array}{cc}
k_{2}^{2} y^{2}+k_{1}^{2} x^{2} & -k_{2}^{2} x y+k_{1}^{2} x y \\
-k_{2}^{2} x y+k_{1}^{2} x y & k_{2}^{2} x^{2}+k_{1}^{2} y^{2}
\end{array}\right] .
$$

The eigenvalues and corresponding eigenvectors are $\tilde{\lambda}_{1}=$ $k_{1}^{2}\left(x^{2}+y^{2}\right)$ with $\tilde{\mathbf{v}}_{\mathbf{1}}=\left(x / \sqrt{x^{2}+y^{2}}, y / \sqrt{x^{2}+y^{2}}\right)$ and $\tilde{\lambda}_{2}=k_{2}^{2}\left(x^{2}+y^{2}\right)$ with $\tilde{\mathbf{v}}_{\mathbf{2}}=\left(-y / \sqrt{x^{2}+y^{2}}, x / \sqrt{x^{2}+y^{2}}\right)$.

In contrast to the eigendirections, the eigenvalues depend on the Jacobian of our transformation, since the nonlinear transformation from polar to Cartesian coordinates changes the metric. According to Append. A the eigenvalues in polar coordinates are $\lambda_{i}=\lambda_{i} / s_{i}^{2}$ for $i=1,2$ with $s_{1}^{2}=$

$\left(\frac{\partial x}{\partial r}\right)^{2}+\left(\frac{\partial y}{\partial r}\right)^{2}=1$ and $s_{2}^{2}=\left(\frac{\partial x}{\partial \theta}\right)^{2}+\left(\frac{\partial y}{\partial \theta}\right)^{2}=r^{2}$. The eigenvalues of our system in Cartesian coordinates are shown as solid and dashed lines in Fig. 2 and a full derivation for the eigenvalues in different coordinate systems is given in Append. A for the general $N$-dimensional case.

Notice that, if we determine the eigenvalues in a Cartesian system, the metric is Euclidean, i.e. the eigenvalues measured in the same units in both $x$ - and $y$-direction directly characterize the diffusion in principal directions. Nonlinear transformations of coordinates such as the one from Cartesian to polar coordinates, however, generally change the metric. In such a system the direction of the maximal eigenvalue is not necessarily the direction with the highest diffusion. For example, in the Hopf-bifurcation in Eqs. [8], the maximal eigenvalue for $r<k_{2} / k_{1}$ is in azimuthal direction, but the maximal diffusion is still in the radial direction.

Looking to $x$ and $y$ as measured variables, they define our Cartesian system and therefore $\tilde{\lambda}_{1}$ is our maximal eigenvalue and $\tilde{\lambda}_{2}$ is associated with the eigendirection showing minimal stochastic fluctuation.

We proceed to verify this result by numerically analyzing the time series $x$ and $y$ plotted in Fig. 3, following the procedure described in the previous section. The procedure is as follows. First, we compute the time series according to Eq. (8) in coordinates $r$ and $\theta$. Then, we transfer this time series to Cartesian coordinates $x, y$. In the new coordinates we calculate the drift vectors $\tilde{\mathbf{D}}^{(1)}$ and diffusion matrices $\tilde{\mathbf{D}}^{(2)}$. Finally, having matrices $\tilde{\mathbf{D}}^{(2)}$ at each mesh point, one easily computes the eigenvalues and eigenvectors, as shown in Fig. 2. The analytical results given by Eq. (10) are reproduced nicely: the stochastic contribution has two eigendirections, one in the radial direction and another in the angular one; the larger eigenvalue is associated with the radial direction. The deviations observed for the minimum eigenvalue occur due to differences in the quality of statistics in different regions of phase space (value of $r$ ): in particular regions in phase space at intermediate values of $r$ are less frequently realized in the data series than others, resulting in a reduced accuracy of the estimated eigenvalues in these regions.

Figures $4 \mathrm{a}-\mathrm{c}$ show the components of the diffusion matrix, namely $\tilde{D}_{11}^{(2)}, \tilde{D}_{12}^{(2)}$ and $\tilde{D}_{22}^{(2)}$ as a function of both $x$ and $y$. Notice that the diffusion matrix is symmetric (see Eq. (3) ) and therefore $\tilde{D}_{21}^{(2)}=\tilde{D}_{12}^{(2)}$. Once the coefficients are numerically derived, one can access their functional behaviour and choose a proper basis of functions to fit the data. In the case of Fig. 4 
(a)

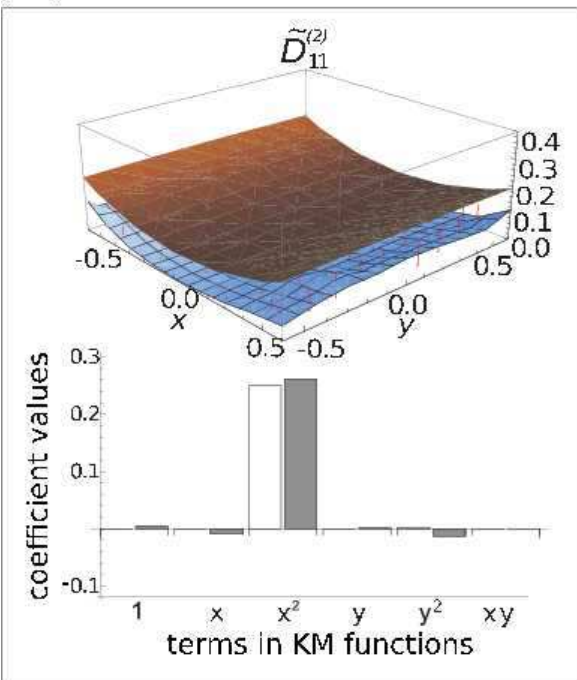

(d)

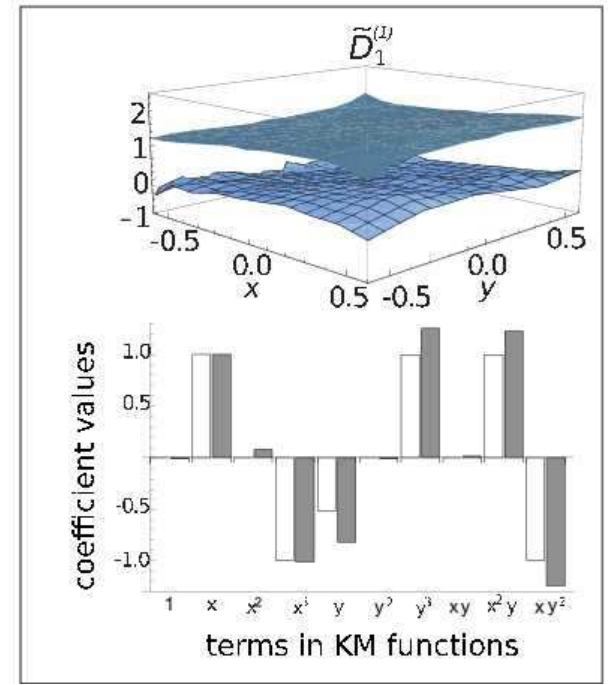

(b)

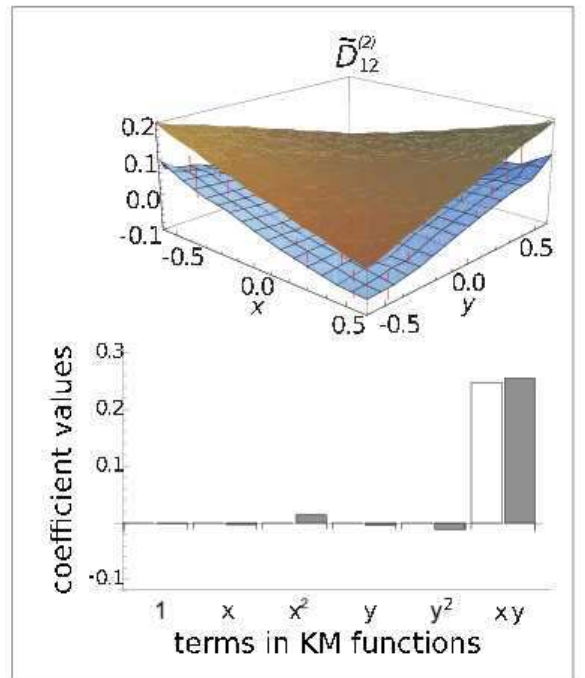

(c)

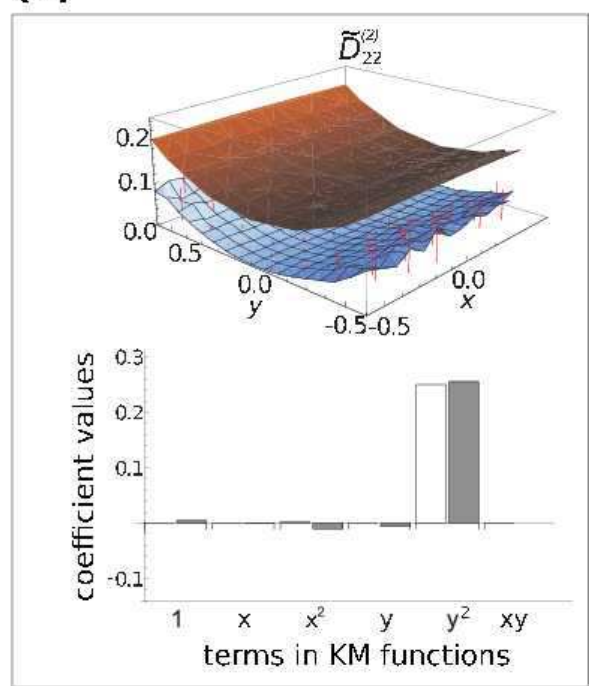

\section{(e)}

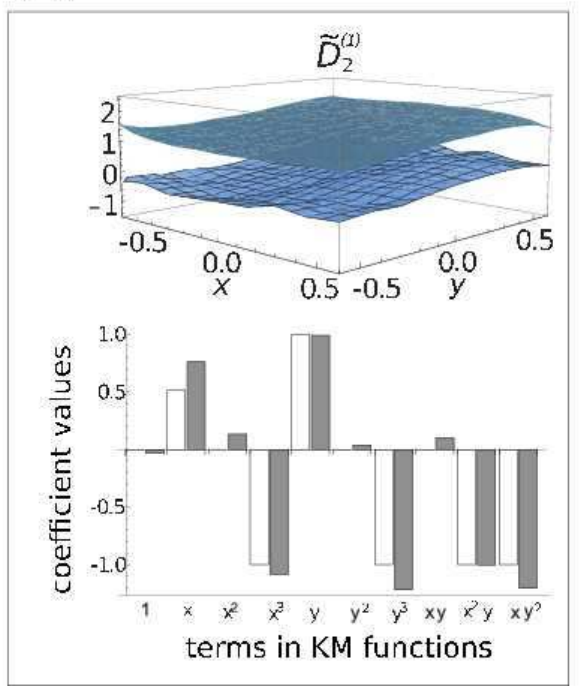

FIG. 4: (Color online) Drift and diffusion functions for the system in Eq. 80 for the same conditions as in Fig. 2 obtained from analysis of the time series $x=r \cos \theta$ and $y=r \sin \theta$. For each drift and diffusion function the bottom surface reflect the numerical results, while the corresponding fitted surface on top is shifted upwards for clarity: (a) $\tilde{D}_{11}^{(2)}(x, y)$, (b) $\tilde{D}_{12}^{(2)}(x, y)$, (c) $\tilde{D}_{22}^{(2)}(x, y)$, (d) $\tilde{D}_{1}^{(1)}(x, y)$, (e) $\tilde{D}_{2}^{(1)}(x, y)$. The bar plots compare the coefficients that fit each function (dark bars) with the analytic, true ones (white bars) (see Tab. I).

the surfaces obtained numerically are fitted to a full quadratic polynomial in $x$ and $y$ through a least square procedure. The coefficients of these polynomial fits are also shown in Fig 4 ac. The results of Fig. $4 \mathrm{a}-\mathrm{c}$ comply with the analytical expression in Eq. 10]. A similar analysis is done for the drift vector, i.e. for the function $\tilde{D}_{1}^{(1)}$ and $\tilde{D}_{2}^{(1)}$, shown in Figs. 4 d-e. Table \lists the values obtained for the coefficients of the surface fits in Fig. 4

It is worth stressing that the aforementioned procedure can likewise be applied to measured multi-dimensional time series in cases where no additional information on the underlying dynamics is available. In this case the corresponding fields of eigenvectors indicate the directions with largest and smallest stochastic contributions. Using this information, a transform of variables to a coordinate system aligned with the direction of the smallest stochastic contribution can be applied, which in the present case would be the tangential direction. In the representation of these new coordinates, the stochastic contribution will then be reduced.

In the previous case, the two eigenvectors for diffusion were introduced tangentially and perpendicularly to the limit cycle. If the principal axes for the stochastic contribution are not aligned with the trajectories of the deterministic part of the system, one might suspect an interplay between both stochastic contribution and the drift of the system.

In order to demonstrate the wider applicability of our 
(a)

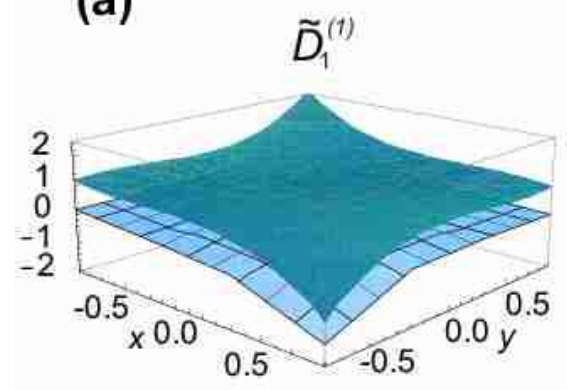

(d)

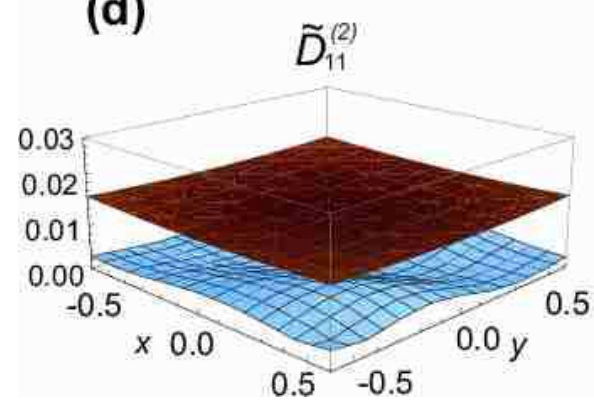

(b)

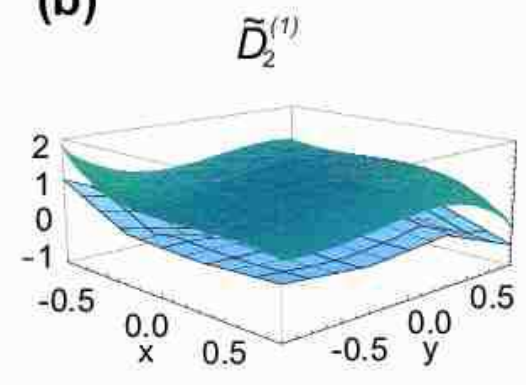

(e)

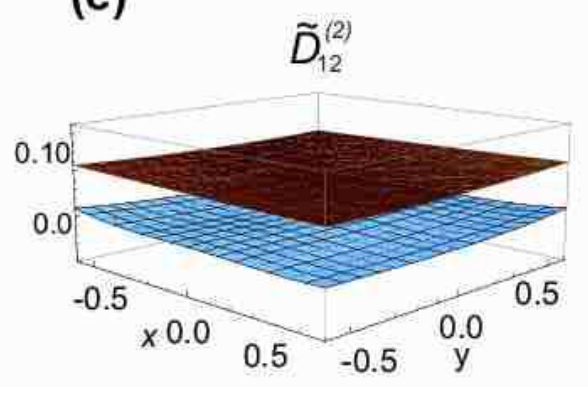

(c)

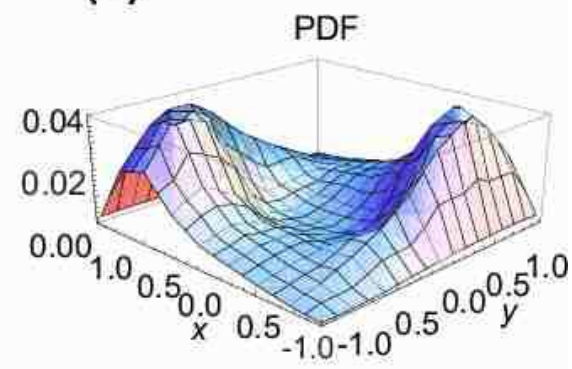

(f)

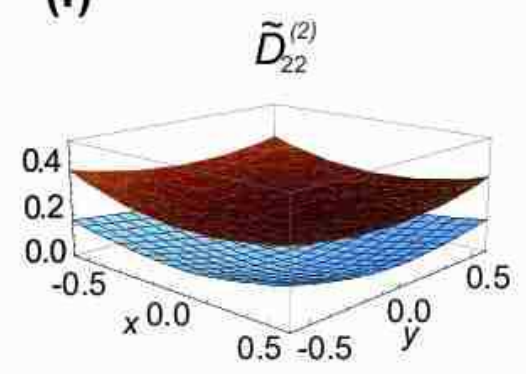

FIG. 5: (Color online) Hopf-system with strong stochastic contribution along the $y$-direction. Drift functions are (a) $\tilde{D}_{1}^{(1)}(x, y)$ and (b) $\tilde{D}_{2}^{(1)}(x, y)$. (c) Probability density function of $(x, y)$ shows the most visited regions in phase space. Diffusion functions are (d) $\tilde{D}_{11}^{(2)}(x, y)$, (e) $\tilde{D}_{12}^{(2)}(x, y)=D_{21}^{(2)}(x, y)$ and (f) $\tilde{D}_{22}^{(2)}(x, y)$. Results were obtained by analyzing the time series $x=r \cos \theta$ and $y=r \sin \theta$ according to Eq. (11) with the same upward shifting for clarity as in Fig. 4 The probability density function in (c) explains the deviations observed for the diffusion functions (see text).

method, we next consider such a case, with the major axis aligned with $y$-direction. We therefore investigate the system

$$
\begin{aligned}
& \frac{d r}{d t}=r\left(1-r^{2}\right)+K_{1} r \cos \theta \Gamma_{1}+K_{2} r \sin \theta \Gamma_{2} \\
& \frac{d \theta}{d t}=\left(\alpha-r^{2}\right)-K_{1} \sin \theta \Gamma_{1}+K_{2} \cos \theta \Gamma_{2} .
\end{aligned}
$$

In Cartesian coordinates the dynamics are described by

$$
\begin{aligned}
& \frac{d x}{d t}=\tilde{h}_{1}+K_{1} r \Gamma_{1} \\
& \frac{d y}{d t}=\tilde{h}_{2}+K_{2} r \Gamma_{2} .
\end{aligned}
$$

with

$$
\begin{aligned}
\tilde{h}_{1}= & x\left(1-\left(x^{2}+y^{2}\right)\right)-y\left(\alpha-\left(x^{2}+y^{2}\right)\right) \\
& +\frac{x}{x^{2}+y^{2}}\left(\left(K_{1}^{2}-2 K_{2}^{2}\right) y^{2}-k_{2}^{2} x^{2}\right) \\
\tilde{h}_{2}= & y\left(1-\left(x^{2}+y^{2}\right)\right)-x\left(\alpha-\left(x^{2}+y^{2}\right)\right) \\
& +\frac{y}{x^{2}+y^{2}}\left(\left(K_{2}^{2}-2 K_{1}^{2}\right) x^{2}-k_{1}^{2} y^{2}\right) .
\end{aligned}
$$

Here we chose $K_{1}=0.05$ and $K_{2}=0.5$. In other words, the impact of stochastic fluctuations is large in the $y$-direction and small along the $x$-axis. Notice that the determinant of the diffusion matrix is not preserved, since the Jacobian of the transformation is not the identity matrix (see Append. $\mathrm{A}$ ).
As already done in the previous example, we integrate system (11) numerically and analyze the resulting data series of $x$ and $y$ values. As can be seen from Fig. 5, the drift functions $\tilde{h}_{1}$ and $\tilde{h}_{2}$ are properly derived (see Figs. 5 a and $5 \mathrm{~b}$ ), as well as the terms of the diffusion matrices $\tilde{D}^{(2)}$ (Figs. 5 5 d-f). The deviations observed for the small $\tilde{D}_{11}^{(2)}$ at particular regions of phase space (Fig. $5 \mathrm{~d}$ ) are due to the lack of accurate statistics at those regions (see the PDF in Fig. 55).

In both cases presented above the estimates of diffusion matrices are in better agreement with the analytical results than the estimates of the corresponding drift vectors. This is a general drawback of the estimation procedure which comes into play upon application to high quality data sets available at high sampling frequencies [19]. Whereas the evaluation of the limiting procedure in (2) converges for the diffusion $(k=2)$, the error of the drift estimates $(k=1)$ diverges in the limit $\tau \rightarrow 0$ for data sets of finite size due to the slow convergence of the law of large numbers implicating small but non-zero stochastic contributions of order $\sqrt{\tau}$ to the first conditional moments, $\mathbf{M}^{(1)}[2]$. The drift estimates e.g. could be improved by advanced estimation techniques applicable at finite time increments [9]. Since the method we aim to present, however, is based on the properties of the diffusion matrices only this effort is not required here.

As can be seen in Fig. 6 and 6 , the eigendirections are properly derived as well as the two eigenvalues plotted in 
(a)

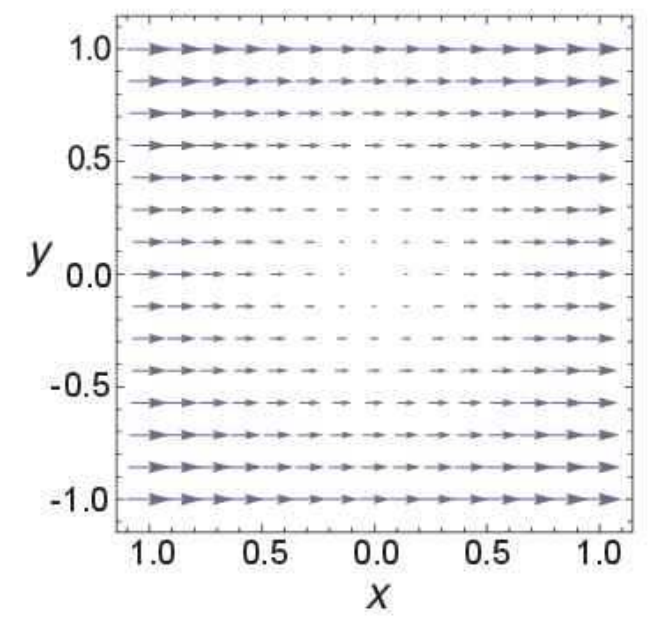

(c)

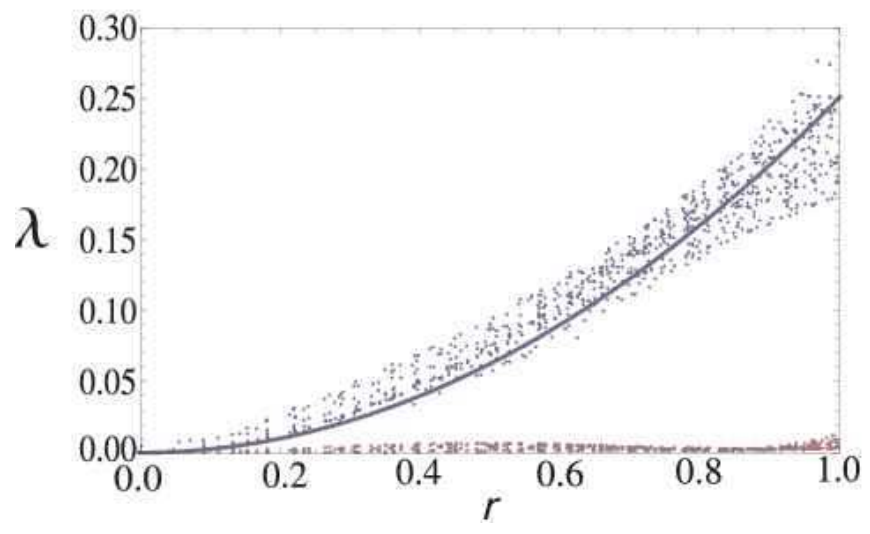

(b)

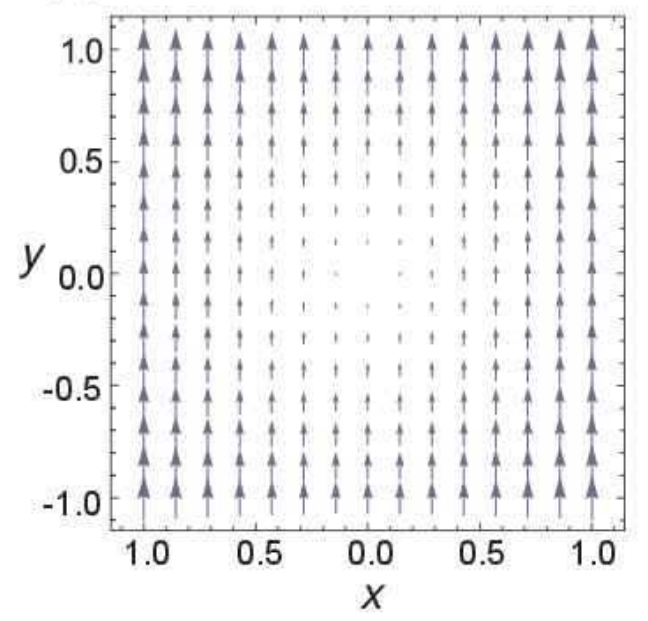

(d)

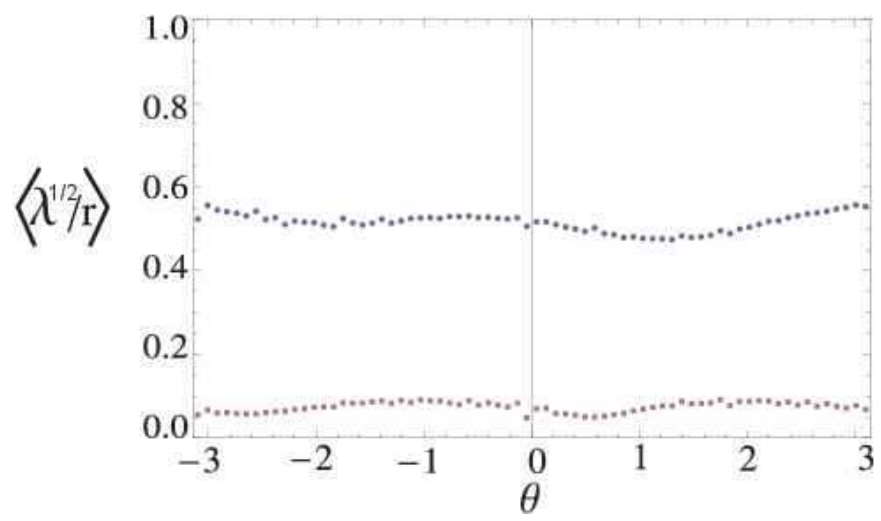

FIG. 6: (Color online) Results of the analysis of diffusion matrices plotted in Fig. 5 obtained from a system with an increased level of dynamical noise on the $y$ component (second example). The individual panels depict (a) the vector fields of eigenvectors for the minimum and (b) the maximum eigenvalue, normalized to the respective eigenvalues, and the dependence of the eigenvalues both both on (c) $r$ and (d) $\theta$.

Fig. 6r and 6 as a function of $r$ and $\theta$, respectively.

With these two examples we first have illustrated that our numerical approach succeeds in estimating the drift and the diffusion functions contributions in a two-dimensional stochastic system. A straightforward computation of the eigenvectors and eigenvalues of the diffusion matrices then enables us to ascertain a set of principal stochastic directions. We would like to note that the method described here bears some resemblance to the well-known principal component analysis[20]. In contrast to principal component analysis performed on the distribution of the measured data the method we propose, however, focuses on the stochastic fluctuations in time. The properties of these fluctuations in general are dependent on the position $X$ resulting in vector fields of the principal axes of the stochastic contributions to the dynamics of the system of consideration. The two methods seem however related and should provide complementary insights when applied to specific sets of empirical data. Such matters are beyond the scope of this paper and will be addressed elsewhere.

We also emphasize that the minimal eigenvalue of the diffusion matrix not necessarily points towards the direction the systems as a whole can be expected to evolve to. It rather reflects the direction of minimal stochastic forcing only. For prediction of the system's evolution in general time propagators need to be taken into account which involve both the stochastic (diffusion) and the deterministic (drift) parts of the dynamics [2]. Nevertheless, being able to ascertain the directions in phase space where fluctuations are weaker enables one to choose a transformation such that part of the new variables have small stochastic terms, reducing the number of variables which are affected by stochastic forces. Further, this is also of physical interest to see in which variables noise is acting, for example to which variable a thermal bath is physically connected, and it may be of technical interest to better detect a 
noise source, for example in an electric circuit [21].

\section{DISCUSSION AND CONCLUSIONS}

In this paper we introduce the concept of eigendirections for the stochastic dynamics in systems of arbitrary dimension. The procedure builds on the modeling of complex systems by means of drift an diffusion functions, that specify a system of coupled Langevin equations. Estimates for these functions can be obtained directly from measurements on the systems without prior knowledge on its dynamics following an approach described in Ref. [1, 2].

In Langevin systems the stochastic forcing is composed of independent Gaussian $\delta$-correlated stochastic fluctuations. The way how these fluctuations effect the system depends both on the diffusion matrix and on the coordinate system chosen.

Whereas in former publications much attention has been paid to imperfections of the Langevin process, like measurement noise or correlated noise and finite size sampling [22- -25$]$ here we introduced a method which allows us to determine the eigendirections along which each stochastic force acts. Each direction of forcing is defined through the eigenvector and the corresponding eigenvalue accounting for its amplitude. The set of eigenvectors does not depend on the choice of the coordinate system and is therefore characteristic for the system. Further, for the particular case where a number $k$ of eigenvalues are negligible in comparison with the others at each mesh point, the number of stochastic variables can be reduced. Even in cases where the number of stochastic variables cannot be reduced, the eigenvector associated with the lowest eigenvalues at each point in phase space indicates the path with minimal stochastic forcing. In any case a transform to new coordinates in the directions of minimum stochastic forcing can be performed, increasing the relative amplitude of the deterministic components and the predictability of the corresponding variable.

The method was successfully applied to a two-dimensional system exhibiting a Hopf-bifurcation. For the Hopfbifurcation the diffusion matrix is better estimated through our analysis than the corresponding drift vector, contrary to what is known in many other situations [2]. One note on the dimensionality of each variable in the set of variables considered must be stressed: If, instead of two position variables, one choses one position and one velocity or acceleration some caution must be given to the dimensionality of the corresponding Kramers-Moyal coefficients.

For each variable value, Kramers-Moyal coefficients are estimated from linear least square fits of the conditional moments as functions of the time increment within an interval of time increments $\Delta t<\tau_{\max }[7]$. Consequently, the errors of both first and second Kramers-Moyal coefficients associated to the finite-time estimation is of the order of the correlation coefficient of that least square fit. Therefore, when computing the eigenvalues of the diffusion matrix, there is typically not a significant error propagation and consequently the same holds for the eigenvectors. Our simulations showed that the relative errors from finite-time estimation and from the least square fit are together typically between $5 \%$ and $10 \%$.

Nevertheless, compared to previous methods for minimization of stochasticity introduced in Refs. [3, 26], our approach has the advantage of not requiring a parametrized Ansatz for quantifying the respective stochastic contributions to the system. Moreover, it should be applicable even in the case where the data sets are contaminated with measurement noise [6].

\section{Acknowledgements}

The authors thank Bernd Lehle for useful discussions. VVV, FR (SFRH/BPD/65427/2009) and PGL (Ciência 2007) thank Fundação para a Ciência e a Tecnologia (FCT) for financial support. All authors thank DAAD and FCT for financial support through the bilateral cooperation DREBM/DAAD/03/2009.

\section{Appendix A: The diffusion matrix in an arbitrary coordinate system}

Consider a transformation of variables $\mathbf{X}=\left\{X_{i}\right\} \rightarrow \tilde{\mathbf{X}}=$ $\left\{\tilde{X}_{i}\right\}$ with $i=1, \ldots, N$, which is given by a two-times continuously differentiable deterministic vector function $\mathbf{F}$

$$
\tilde{\mathbf{X}}=\mathbf{F}(\mathbf{X}, t)
$$

Using Itô's formula [12, 13], a truncated form of the Itô Taylor expansion, the Langevin equations for the new variables take the form

$$
\begin{aligned}
d \tilde{X}_{i}= & \frac{\partial F_{i}}{\partial t} d t+\sum_{k=1}^{N} \frac{\partial F_{i}}{\partial X_{k}} d X_{k}+ \\
& \frac{1}{2} \sum_{k=1}^{N} \sum_{l=1}^{N} d X_{k} \frac{\partial^{2} F_{i}}{\partial X_{k} \partial X_{l}} d X_{l}+\ldots .
\end{aligned}
$$

Eq. (4) can be rewritten to

$$
d X_{i}=D_{i}^{(1)}(\mathbf{X}) d t+\sum_{j=1}^{N} g_{i j}(\mathbf{X}) \Gamma_{j}(t) \sqrt{d t} .
$$

Inserting this expression into Eq. A2 , retaining all terms in the expansion up to order $d t$ while neglecting terms of order $(d t)^{3 / 2}$ or higher, and taking advantage of the statistics of the stochastic forcing, Eq. (5), one obtains for the third expression on 
the r.h.s.

$$
\frac{1}{2} \sum_{k=1}^{N} \sum_{l=1}^{N}\left(D_{k}^{(1)} d t+\sum_{j=1}^{N} g_{k j} \Gamma_{j} \sqrt{d t}\right) \frac{\partial^{2} F_{i}}{\partial X_{k} \partial X_{l}}\left(D_{l}^{(1)} d t+\sum_{m=1}^{N} g_{l m} \Gamma_{m} \sqrt{d t}\right)=\frac{1}{2} \sum_{k=1}^{N} \sum_{l=1}^{N} \sum_{j, m=1}^{N} 2 g_{k j} g_{l m} \frac{\partial^{2} F_{i}}{\partial X_{k} \partial X_{l}} \delta_{j m} d t
$$

Thus, the transformed Langevin equation has the form

$$
d \tilde{X}_{i}=\frac{\partial F_{i}}{\partial t} d t+\sum_{k=1}^{N} \frac{\partial F_{i}}{\partial X_{k}}\left(D_{k}^{(1)} d t+\sum_{j=1}^{N} g_{k j} \Gamma_{j}(t) \sqrt{d t}\right)+\sum_{k=1}^{N} \sum_{l=1}^{N} \sum_{j=1}^{N} g_{l j} g_{k j} \frac{\partial^{2} F_{i}}{\partial X_{k} \partial X_{l}} d t+\mathcal{O}(d t)^{3 / 2}
$$

Restricting to stationary processes $\left(\frac{\partial F_{i}}{\partial t}=0\right)$ and using the notation

$$
\frac{d \tilde{X}_{i}}{d t}=\tilde{D}_{i}^{(1)}(\tilde{\mathbf{X}})+\sum_{j=1}^{N} \tilde{g}_{i j}(\tilde{\mathbf{X}}) \Gamma_{j}(t)
$$

the transformed drift function reads

$$
\tilde{D}_{i}^{(1)}=\sum_{k=1}^{N}\left(D_{k}^{(1)} \frac{\partial F_{i}}{\partial X_{k}}+\sum_{l=1}^{N} \sum_{j=1}^{N} g_{l j} g_{k j} \frac{\partial^{2} F_{i}}{\partial X_{k} \partial X_{l}}\right)
$$

The components of matrix $\mathbf{G}$ transform as

$$
\tilde{g}_{i j}=\sum_{k=1}^{N} g_{k j} \frac{\partial F_{i}}{\partial X_{k}} \quad \Leftrightarrow \quad \tilde{\mathbf{G}}=\mathbf{J G},
$$

where $\mathbf{J}$ is the Jacobian of our transformation from coordinates $X_{i}$ (vector basis $\mathbf{e}_{i}$ ) to $\tilde{X}_{i}$ (vector basis $\tilde{\mathbf{e}}_{i}$ ).

From elementary algebra it is known that a vector $\mathbf{v}$ can be written in both coordinate systems:

$$
\begin{aligned}
\mathbf{v} & =\sum_{j} \tilde{v}_{j} \tilde{\mathbf{e}}_{j}=\sum_{j} \tilde{v}_{j} \sum_{k} J_{j k} \mathbf{e}_{k} \\
& =\sum_{k}\left(\sum_{j} J_{j k} \tilde{v}_{j}\right) \mathbf{e}_{k}=\sum_{k}\left(\sum_{j} J_{k j}^{T} \tilde{v}_{j}\right) \mathbf{e}_{k} \\
& \equiv \sum_{k} v_{k} \mathbf{e}_{k} .
\end{aligned}
$$

Thus, the matrix $\tilde{\mathbf{U}}$ incorporating the columns of eigenvectors $\mathbf{u}_{k}$ of matrix $\tilde{\mathbf{G}}$, with coordinates in basis $\tilde{\mathbf{e}}_{i}$, can be written as $\mathbf{U}=\mathbf{J}^{\mathrm{T}} \tilde{\mathbf{U}}$ with $\mathbf{U}=\left[\begin{array}{llll}\mathbf{u}_{1} & \mathbf{u}_{2} & \ldots & \mathbf{u}_{N}\end{array}\right]$ fulfilling $\mathbf{U}^{-1}=\mathbf{U}^{T}$. Thus, from Eq. (A8) one obtains

$$
\tilde{\mathbf{U}}^{T} \tilde{\mathbf{G}} \tilde{\mathbf{G}}^{T} \tilde{\mathbf{U}}=\mathbf{U}^{T} \mathbf{G G}^{T} \mathbf{U}
$$

So, the eigenvectors of $\mathbf{D}^{(2)}$ and $\tilde{\mathbf{D}}^{(2)}$ are the same, apart from the basis in which they are considered, and in the original (Cartesian) coordinate system $\mathbf{X}$, the transformation to principal axes is given by Eq. (A10) i.e.

$$
\mathbf{U}^{T} \mathbf{D}^{(2)} \mathbf{U}=\operatorname{diag}\left[\begin{array}{llll}
\lambda_{1} & \lambda_{2} & \ldots & \lambda_{N}
\end{array}\right]
$$

At regular points the transformation in Eq. (A1) can be inverted,

$$
\mathbf{X}=\mathbf{F}^{-1}(\tilde{\mathbf{X}})=: \mathbf{f}(\tilde{\mathbf{X}})
$$

By definition $\mathbf{f}(\tilde{\mathbf{X}})$ is chosen such that the normalized eigenvectors are given by

$$
\mathbf{u}_{k}=\frac{1}{s_{k}} \frac{\partial \mathbf{f}}{\partial \tilde{X}_{k}} \quad \text { with } \quad s_{k}=\left|\frac{\partial \mathbf{f}}{\partial \tilde{X}_{k}}\right| .
$$

The Jacobian of $\mathbf{f}(\tilde{\mathbf{X}})$ can then be written as

$$
\tilde{J}_{i k}(\tilde{\mathbf{X}})=\frac{\partial f_{i}}{\partial \tilde{X}_{k}}
$$

or in a more convenient form

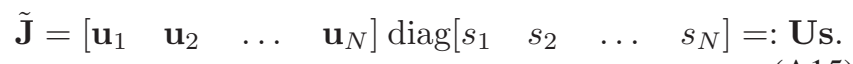

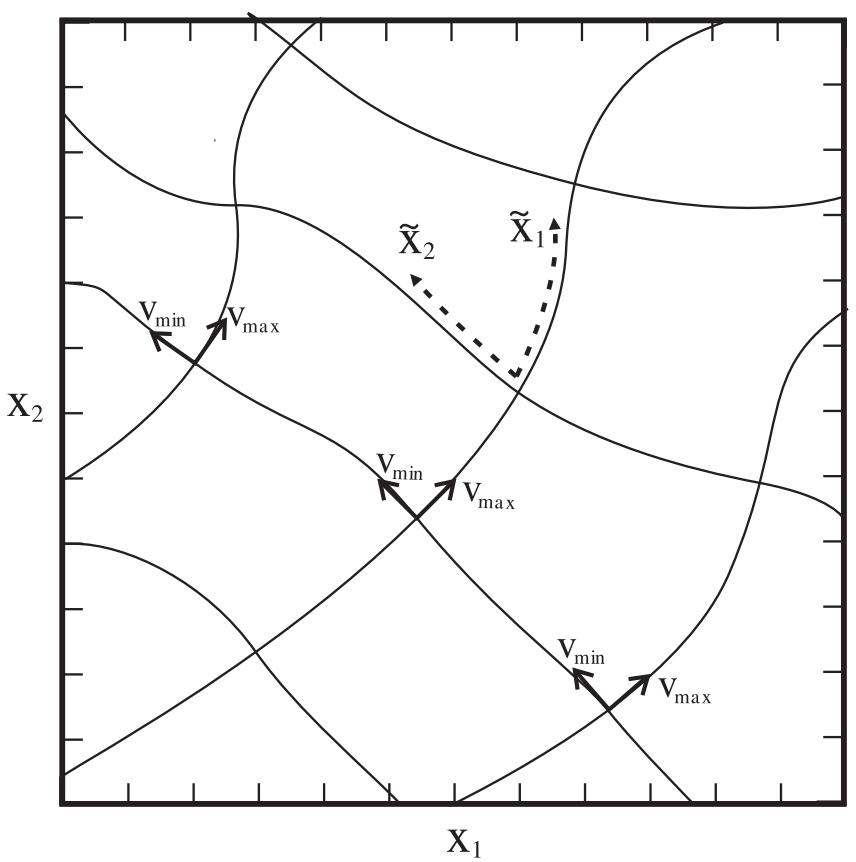

FIG. 7: Interchanging between coordinate systems. Arrows indicate that eigenvectors of the eigenvalues of the diffusion matrix (see Eq. A19) at each point of the mesh from which drift and diffusion coefficients are derived. Here the two-dimensional case is illustrated. Extension to arbitrary number of variables is straightforward.

The diagonal matrix s describes the metric of the new system. The Jacobian of the transformation in Eq. A1] is the 
inverse relation

$$
\mathbf{J}=\mathbf{s}^{-1} \mathbf{U}^{T}
$$

Introducing this relation into Eq. A8 yields

$$
\tilde{\mathbf{G}}=\mathbf{J G}=\mathbf{s}^{-1} \mathbf{U}^{T} \mathbf{G}
$$

i.e. the transformed diffusion matrix reads

$$
\tilde{\mathbf{D}}^{(2)}=\tilde{\mathbf{G}} \tilde{\mathbf{G}}^{T}=\mathbf{s}^{-1} \mathbf{U}^{T} \mathbf{G G}^{T} \mathbf{U} \mathbf{s}^{-1} .
$$

Inserting Eqs. A11 and A15 finally leads to the diagonal matrix

$$
\tilde{\mathbf{D}}^{(2)}=\operatorname{diag}\left[\begin{array}{llll}
\frac{\lambda_{1}}{s_{1}^{2}} & \frac{\lambda_{2}}{s_{2}^{2}} & \ldots & \frac{\lambda_{N}}{s_{N}^{2}}
\end{array}\right] .
$$

In practice, we have no access to the transformation in Eq. A1. Instead we have a grid (numerical) representation in $\mathbf{X}$ coordinate system and search for the transformation in Eq. A1 is such that one of the new variables, say $\tilde{X}_{1}$, is defined by the field of eigenvectors $\mathbf{u}_{1}$ corresponding to the maximal eigenvalue $\lambda_{1}$. Then, each orthogonal direction is consequently defined by one of the other (orthogonal) eigenvectors $\mathbf{u}_{j}$ with $j=1, \ldots, N$. Figure 7 illustrates this for the two-dimensional case.

To obtain the coordinates of each point $P$ in this grid in the new coordinates $\tilde{\mathrm{X}}$ one considers the transformation A1 together with the inverted transformation $\mathrm{A} 12$ and solves the system of PDEs in A13. The problem lies in the coupling introduced by the factors $s_{k}$ and the additional complication of finding the correct boundary conditions which turns the solution of the PDEs into a complicated problem, even numerically, although in 2D the $s_{k}$ can be neglected as we are only interested in finding the direction $\frac{\partial f_{i}}{\partial X_{k}}$. In most cases, however, it should be possible to guess a suitable transform from visual inspection of the fields of eigenvectors. From one point to the next one in the mesh, the eigenvectors are sorted according to continuity arguments. Further, depending on the obtained fields of eigenvectors, a scaled polar form or hyperbolic coordinates may be considered, where the parameters of said transform can then be fitted to the vector fields. Such particular cases depend on the specific data set at hand and will be addressed elsewhere.
[1] R. Friedrich and J. Peinke, Phys. Rev. Lett. 78, 863 (1997).

[2] R. Friedrich, J. Peinke and M.R.R. Tabar, Complexity in the view of stochastic processes in Springer Encyclopedia of Complexity and Systems Science (Springer, Berlin, 2008).

[3] P.G. Lind, A. Mora, J.A.C. Gallas and M. Haase, Phys. Rev. E 72, 056706 (2005).

[4] R. Friedrich, J. Peinke and Ch. Renner, Phys. Rev. Lett. 84, 5224 (2000).

[5] F. Ghasemi, M. Sahimi, J. Peinke, R. Friedrich, G.R. Jafari and M.M.R. Tabar, Phys. Rev. E 75, 060102 (2007).

[6] F. Böttcher, J. Peinke, D. Kleinhans, R. Friedrich, P.G. Lind, M. Haase, Phys. Rev. Lett. 97090603 (2006).

[7] P.G. Lind, M. Haase, F. Böttcher, J. Peinke, D. Kleinhans and R. Friedrich, Phys. Rev. E 81041125 (2010).

[8] J. Carvalho, F. Raischel, M. Haase and P.G. Lind, J. Physics. Conf. Ser. 285012007 (2011).

[9] D. Kleinhans, R. Friedrich , and A. Nawroth, Phys. Lett A, 346 42-46 (2005).

[10] G. Gottschall, M. Wächter and J. Peinke, New J. Phys. 10(8) 083034 (2008).

[11] S.J. Lade, Phys. Lett. A 373 3705-3709 (2009).

[12] H. Risken, The Fokker-Planck Equation, (Springer, Heidelberg, 1984).

[13] C. W. Gardiner, Handbook of stochastic Methods, (Springer, Germany, 1997).

[14] D. Lamouroux and K. Lehnertz, Phys. Lett. A 373 3507-3512 (2009).

[15] J. Gradis̃ek, R. Friedrich, E. Govekar and I. Grabec, Meccanica, 38, 33 (2003).
[16] A. M. van Mourik, A. Daffertshofer, and P. J. Beek, Biological cybernetics 94, 233 (2006).

[17] J. Argyris, G. Faust, M. Haase and R. Friedrich, Die Erforschung des Chaos (Springer, Berlin, 2010).

[18] N.G. van Kampen, Stochastic Processes in Physics and Chemistry (North Holland, 1992).

[19] D. Kleinhans and R. Friedrich, in Wind Energy: Proceedings of the Euromech Colloquium, Eds. J. Peinke, P. Schaumann, and S. Barth (Springer, Berlin, 2007), pp. 129-133.

[20] H. Kantz and T. Schreiber, Nonlinear time series analysis Cambridge Univ. Press, Cambridge (1997)

[21] R. Friedrich, S. Siegert, J. Peinke, St. Lück, M. Siefert, M. Lindemann, J. Raethjen, G. Deuschl, G. Pfister, Phys. Lett. A 271 217-222 (2000).

[22] M. Ragwitz and H. Kantz, Phys. Rev. Lett. 87, 254501 (2001)

[23] R. Friedrich, C. Renner, M. Siefert and J. Peinke, Phys. Rev. Lett. 89, 149401 (2002).

[24] D. Kleinhans and R. Friedrich, Physics Letters A 80, 368 (2007).

[25] C. Anteneodo and R. Riera, Phys. Rev. E 80, 031103 (2009).

[26] P.G. Lind, A. Mora, M. Haase and J.A.C. Gallas, Int. J. Bif. Chaos 17(10) 3461-3466 (2007).

[27] A.M. van Mourik, A. Daffertshofer and P.J. Beek, Phys. Lett. A 351(1-2) 13 (2006).

[28] In practice binning or kernel based approaches with a certain threshold are applied in order to evaluate the condition $\mathbf{Y}(t)=$ X. See e.g. Ref. [14] for details. 